\title{
FURTHER MODELS OF PLANETARY NEBULA SPECTRAL EVOLUTION
}

\author{
K. VOLK \\ Department of Physics and Astronomy, University of Calgary, 2500 University Drive N.W., \\ Calgary, Alberta, Canada T2N 1 N4
}

\begin{abstract}
Studies of the total spectra of planetary nebulae (PN) show that they usually emit significant fractions of their total energy at infrared wavelengths. Many PN have IRAS observations but we do not understand their broad-band colours. To model these observations requires a combination of a photoionization treatment of the ionized region and a radiative transfer treatment of any neutral dust shell. Including the effects of the central star evolution and an assumed galactic distribution allows many observables to be simulated. I find that the Schönberner 0.64 $\mathrm{M}_{\odot}$ evolutionary track is too slow in the transition to high temperature to match the IRAS colours of PN if a typical AGB wind expansion speed is $10 \mathrm{~km} \mathrm{~s}^{-1}$. Accelerating the evolution track by a factor of 2 produces reasonable agreement. An equal mixture of carbon-rich and oxygen-rich sources with mass loss rates of $2 \times 10^{-5} \mathrm{M}_{\odot} \mathrm{yr}^{-1}$ seems to reasonably match the general properties of the PN data from IRAS although the details are still unclear. These models strongly indicate that the post-AGB luminosity decline deduced by Knapp (1986) from radio CO line observations is not correct.
\end{abstract}

\section{Introduction}

The IRAS satellite observed hundreds of planetary nebulae (PN) at wavelengths from 12 to 100 $\mu \mathrm{m}$. In addition LRS spectra are available for some of the brighter PN in the 8 to $23 \mu \mathrm{m}$ wavelength range. It was surprising to observe so many $\mathrm{PN}$ because the dust was thought to be relatively short-lived within the ionized region due to ionic sputtering. The IRAS observations are not well understood: the LRS spectra and broad-band colours do not seem to indicate a clear evolutionary path and there is a tremendous diversity in the infrared character of PN, much more so than for compact HII region sources. Zhang and Kwok (1991) have shown that many PN emit a large fraction of their energy in the far-infrared, as had previously been observed for smaller groups of PN (i.e. Cohen and Barlow 1974).

To understand these observations better requires a spectral evolution model which includes the evolution of both the nebular spectrum and the thermal dust emission. After the asymptotic giant branch (AGB) evolution ends, the remnant dust shell expands away from the star at constant speed while the star evolves to higher temperature. There may also be a second, fast wind which produces an interacting winds shell. This circumstellar shell is ionized from within by the star. All of these factors must be considered as functions of time. The basic evolution of the spectrum is determined by competition between the central star evolution and the expansion of the dust shell. 


\section{The Model}

A multi-component modelling process is required because of the wide range of physical processes involved in forming the energy distribution. I used the Schönberner central star tracks to follow the central star evolution (although I ended up accelerating the evolution by a factor of 2 as discussed below). For any time after the AGB the Schönberner tracks give the temperature and luminosity of the central star which is assumed to be a blackbody. There were 4 runs for different initial dust shells, corresponding to $\dot{M}$ values of $2.1 \times 10^{-5}$ and $5.2 \times 10^{-6} \mathrm{M}_{\odot} \mathrm{yr}^{-1}$ and either silicate or graphite grains. The dust density distribution was inverse-square until a fast wind begins, when $\mathrm{T}_{*}=10^{4} \mathrm{~K}$, after which a shell + wind density distribution was used.

Once the star becomes hot enough to ionize the surrounding gas, the photoionization program CLOUDY (kindly provided by Gary Ferland) was used to model the ionized gas including an appropriate amount of dust. If the nebula is predicted to be ionization bounded the radius of the ionization front and the output spectrum from CLOUDY were used as inputs to a dust radiative transfer program (DUSTCD, originally by C. M. Leung). There is therefore an inconsistency in the modelling because no dust radiative transfer calculation is done for the ionized region. This type of calculation was carried out for about 120 times during the post-AGB evolution, until the star has passed onto the white dwarf cooling curve at an age of $\approx 10^{4}$ years.

These model runs differ from those in Volk (1992) because the newer version of CLOUDY includes dust in the ionized region, rather than having to carry out the dust radiative transfer independently of the photoionization calculation. A discrepancy between CLOUDY and the DUSTCD radiative transfer program mentioned in Volk (1992) has been resolved, but there still remain some difficulties in combining CLOUDY and DUSTCD results which produce odd effects in certain parts of the model tracks. The process used to convert the spectral evolution results to simulate observations - photometry, $5 \mathrm{GHz}$ radio flux density, angular radii, infrared excess values, and line ratios - is discussed in Volk (1992).

\section{Results Of The New Runs}

Early trial runs indicated that the Schönberner (1983) model tracks evolve too slowly to match the IRAS colours of PN even for the $0.64 \mathrm{M}_{\odot}$ case. This effect is quite pronounced and seems to be a very robust conclusion for an expansion speed of $10 \mathrm{~km} \mathrm{~s}^{-1}$. It was found that the $0.64 \mathrm{M}_{\odot}$ track had to be accelerated by a factor of 2 , at least during the initial temperature rise to $>3 \times 10^{4} \mathrm{~K}$, for the model colour track to "turn back" at the proper $12 / 25 \mu \mathrm{m}$ colour. This can be explained in several ways: the expansion speed could be only $5 \mathrm{~km} \mathrm{~s}^{-1}$, the typical PN central star could be slightly more massive than $0.64 \mathrm{M}_{\odot}$, or possibly there is stronger post-AGB mass loss from the residual envelope than was assumed by Schönberner. As observations of suspected post-AGB stars often show signs of continuing mass loss, I consider the last possibility to be the most likely. A wider range of post-AGB stellar evolution tracks is needed to study this problem, because this difficulty with the original Schönberner tracks has been found before from other studies.

One new result of these models was that the graphite dust runs consistently predict more infrared emission than did the silicate ones. The predicted IRAS $12 \mu \mathrm{m}$ fluxes were generally 1 to 2 magnitudes higher at any given time for the graphite shell. Thus, even if there are not as many carbon-rich PN and PPN as oxygen-rich ones there is a strong observational bias towards the carbon-rich sources using IRAS. This may explain why most of the brighter PN and PPN observed by IRAS appear to be carbon-rich. By contrast optically selected nebulae are then strongly biased against young, carbon-rich nebulae due to the strong dust extinction from the shell, so objects such as IRAS $21282+5050$ or $07027-7934$ were discovered by IRAS.

The runs for the lower $\dot{M}$ values using either type of dust were found to be strongly matterbounded for most of the PN evolution, and the predicted optical line strengths do not match well 
with what is generally observed. Thus these tracks were rejected. For the higher $\dot{M}$ values the nebulae are predicted to be matter-bounded during the phase of maximum ionizing photon flux but ionization-bounded both as young nebulae and once the luminosity of the central star falls below about $10^{3} \mathrm{~L}_{\odot}$. Together the graphite and silicate dust tracks cover the centre of the observed IRAS colours of PN, but the predicted colours do not show nearly as much scatter as is observed. Part of the cause of the scatter may be PAH emission in the carbon-rich nebulae, but clearly more work needs to be done to identify why some observed PN have unusual colours compared with the model tracks.

To show the degree to which the models match the IRAS data, Figure 1 gives the observed brightness distribution of optically identified PN in the 4 IRAS bands along with the model predictions for an equal mix of carbon-rich and oxygen-rich nebulae. A scale factor of 0.73 has been applied to the model curves. A good match in shape and level is obtained at $60 \mu \mathrm{m}$. At 100 $\mu \mathrm{m}$ the data probably suffers completeness problems even at relatively high flux densities, so the observed curve rolls off from the model curve at $\approx 10 \mathrm{Jy}$. At $25 \mu \mathrm{m}$ a good match is obtained for PN brighter than $\approx 2 \mathrm{Jy}$ but thereafter there are no model sources. At $12 \mu \mathrm{m}$ the shape of the model curve is correct but the number of predicted sources is $\approx 10 \%$ too low which may simply mean that the $12 \mu$ m luminosities are $\approx 10 \%$ too low, due to PAH emission in real carbon-rich nebulae for example. Together the agreement at 12 and $60 \mu \mathrm{m}$ and the disagreement at $25 \mu \mathrm{m}$ are rather peculiar and I do not know what the cause of this is. Overall the match is fairly good. The fit varies somewhat for different mixtures of the carbon-rich and oxygen-rich nebulae.

\section{The $\beta$ Problem For PN}

There is a direct conclusion from Figure 1 which has a bearing upon papers in the literature on the radio CO line observations of $\mathrm{PN}$ and PPN. For both AGB and post-AGB sources observed in CO it is customary to calculate a $\beta$ value which is the ratio of the (scalar) momentum carried in the mass-loss to the photon momentum from the star. This value is found to be of order 1 for AGB stars but rises to $\approx 100$ in PN. Knapp (1986) interprets this as a direct result of a luminosity drop by a large factor soon after the AGB, whereas the central star models indicate a fairly constant luminosity during the rise to high temperature. In the context of this paper, that the Schönberner tracks produce a reasonable fit to the IRAS observations for an assumed galactic population of 18000 PN makes Knapp's interpretation difficult to sustain. For if post-AGB sources do indeed fade by a factor of 50, say, soon after the AGB then these models have the young PN too bright by a factor of 50 . To produce the general agreement in infrared brightness - and optical and radio brightness too - with observations would be impossible unless my assumed galactic population is 50 times too low (or the Schönberner AGB luminosity values are a factor of 50 too low, but that would cause further conflicts with observations). However, as bad as PN distance determinations are it is impossible that there could be a total galactic population of about $10^{6} \mathrm{PN}$ and that all those we observe are quite local. Thus I conclude that the change in $\beta$ values do not indicate a luminosity effect even though this is the simplest interpretation.

\section{References}

Cohen, M., and Barlow, M. J. (1974) ApJ, 193, 401.

Knapp, G. R. (1986) ApJ, 311, 731.

Schönberber, D. (1983) ApJ, 272, 708.

Volk, K. (1992) ApJSupp, 80, 347.

Zhang, C.-Y., and Kwok, S. (1991) A\&A, 250, 179. 

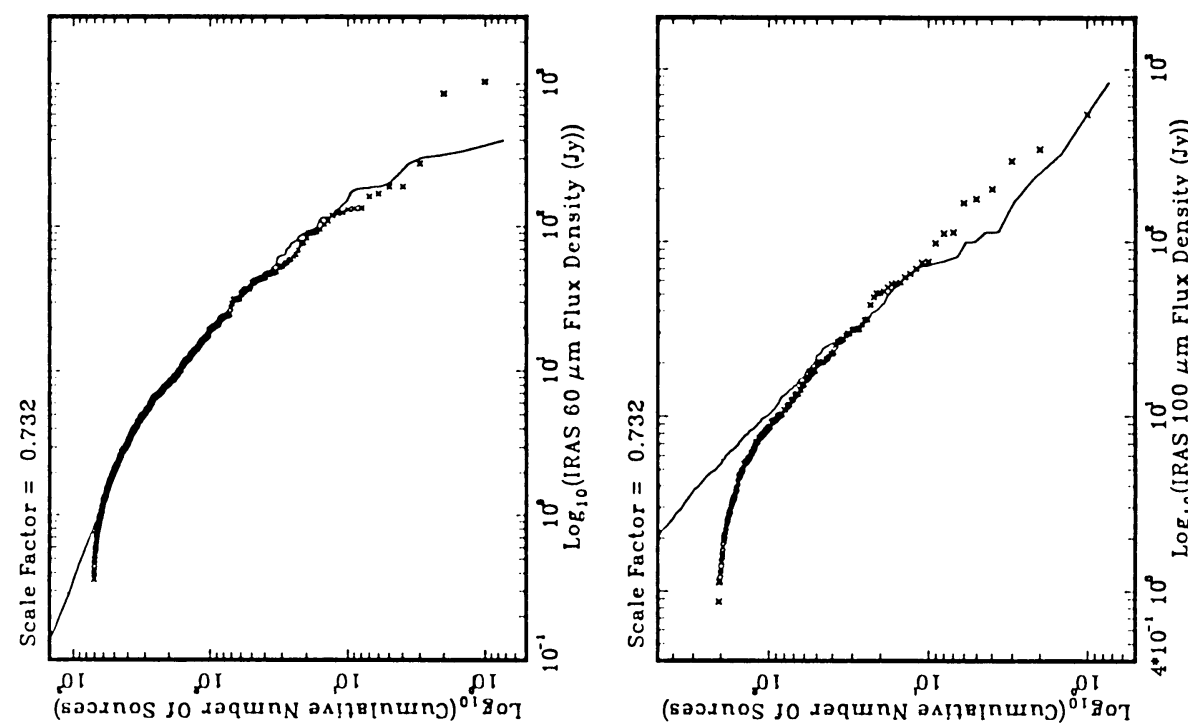

8

ลิ ผ

के ल

范

胥

药 节

\& 0 i

莳

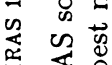

○

๑)

.

-

(saoxnos 70 daqun
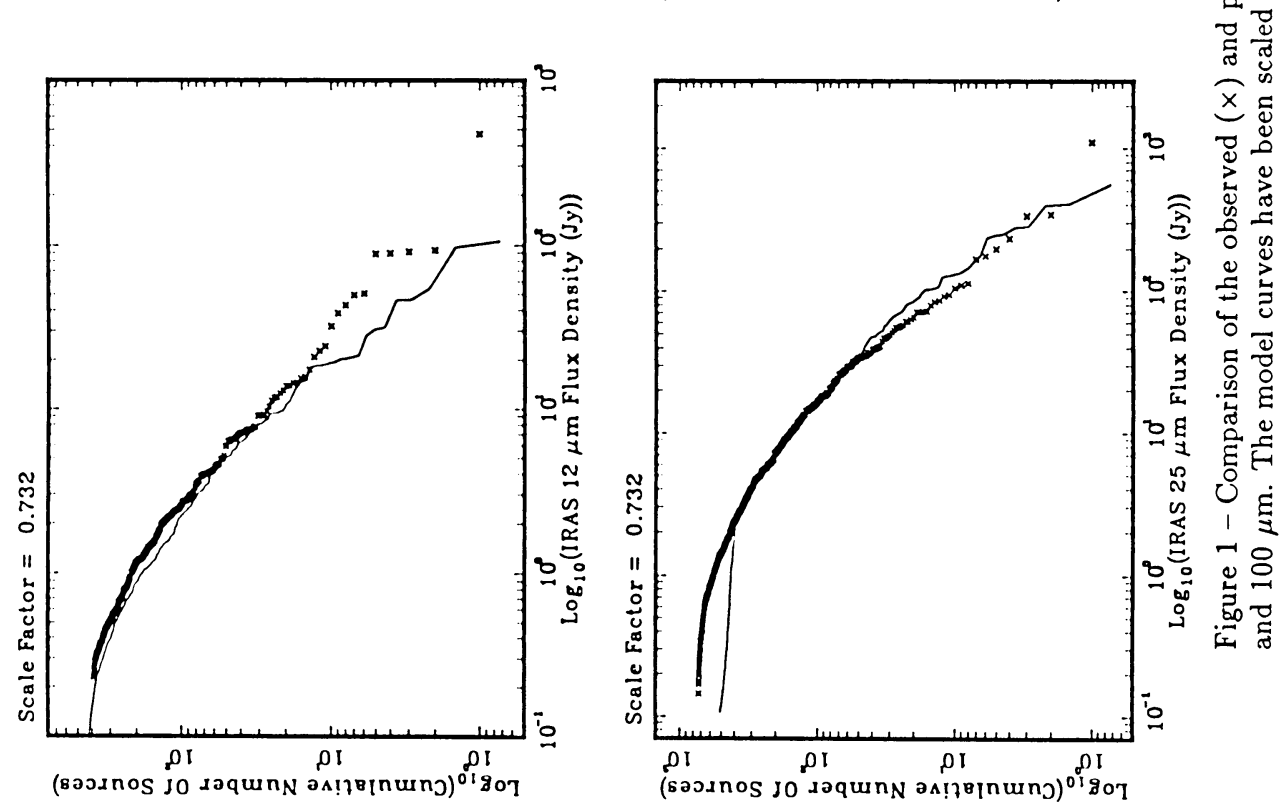